Cipango $\begin{aligned} & \text { Cipango } \\ & \text { Cahiers d'études japonaises }\end{aligned}$

$15 \mid 2008$

Guerre, colonialisme et commémoration

\title{
Le traumatisme du Pacifique
}

Le sanctuaire Yasukuni et sa vision de la guerre

Pacific Trauma: Yasukuni and the Fetishised Narrative of War

\section{John Breen}

Traducteur : Noémi Godefroy

\section{(2) OpenEdition}

\section{Journals}

\section{Édition électronique}

URL : https://journals.openedition.org/cipango/105

DOI : $10.4000 /$ cipango. 105

ISSN : 2260-7706

\section{Éditeur}

INALCO

\section{Édition imprimée}

Date de publication : 1 janvier 2008

Pagination : 65-77

ISBN : 978-2-85831-177-4

ISSN : $1164-5857$

\section{Référence électronique}

John Breen, «Le traumatisme du Pacifique », Cipango [En ligne], 15 | 2008, mis en ligne le 11 novembre 2011, consulté le 30 juin 2021. URL : http://journals.openedition.org/cipango/105 ; DOI : https://doi.org/10.4000/cipango.105

Ce document a été généré automatiquement le 30 juin 2021

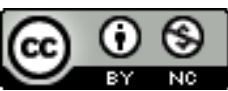

Cipango est mis à disposition selon les termes de la Licence Creative Commons Attribution - Pas d'Utilisation Commerciale 4.0 International. 


\title{
Le traumatisme du Pacifique
}

\author{
Le sanctuaire Yasukuni et sa vision de la guerre \\ Pacific Trauma: Yasukuni and the Fetishised Narrative of War
}

\author{
John Breen \\ Traduction : Noémi Godefroy
}

1 « Un homme m'a dit que vous étiez l'un de ceux qui avaient tiré à la courte paille pour désigner celui qui tuerait Hashimoto Giichi, un homme pour qui j'avais beaucoup de respect... Vous l'avez abattu, puis avez fait passer cela pour une mort naturelle. ». C'est avec ces mots que le caporal Okuzaki Kenzō 奥崎謙三 (1920-2005), anciennement membre du $36^{e}$ corps du Génie, s'adresse avec colère à son ancien officier supérieur, le sergent Yamada, quarante ans après la fin de la guerre. Cette confrontation ouvre le neuvième chapitre du remarquable documentaire de Hara Kazuo 原一男 (né en 1945), Yuki yukite shingun ゆきゆきて神軍 (1987) ${ }^{1}$. Dans celui-ci, on suit les démarches d'okuzaki qui débusque, l'un après l'autre, les officiers et sous-officiers impliqués dans l'exécution de ses compagnons en Nouvelle-Guinée en 1945.

Le chapitre enchaîne sur cet échange bouleversant entre les deux hommes :

Yamada: "Il y a des choses qui sont tout simplement trop douloureuses pour que l'on puisse en parler... Si vous aviez lu ce que j'ai écrit, vous auriez compris ce par quoi je suis passé... Nous mangions des racines, de l'herbe, nous mangions tout ce qui se trouvait entre les racines et la cime des arbres. "

Okuzaki : "Tous ceux qui ont participé à la guerre ont écrit ce genre de choses... Je ne suis pas venu ici pour écouter des banalités."

Yamada: "J'ai décidé de ne rien dire de plus. J'ai simplement été entraîné par mes supérieurs..."

Okuzaki : "Mais bien sûr... Si, comme vous le dites, vous êtes vraiment touché par le sort qu'ont subi vos compagnons disparus, eh bien, parlez! Quel autre moyen avons-nous d'empêcher les hommes de perpétrer de nouveau de telles atrocités? Vous avez connu l'enfer. Comment pouvez-vous prétendre apaiser les âmes des morts de cette guerre sans évoquer cet enfer? Vous gardez le silence par peur de ce que penseront votre femme, vos enfants et vos petits-enfants, c'est ça? J'ai amené ici avec moi le frère de Hashimoto pour qu'il entende la vérité sur ce qui s'est passé. Vous n'avez rien à dire? Vous n'allez pas vous excuser?"

Yamada : «t pourquoi devrais-je m'excuser?» 
Okuzaki : «Pour les vies que vous avez fauchées, voilà pourquoi. »

Yamada: "Je ne sais pas de quoi vous parlez..."

Okuzaki : "Je veux connaître les faits. Seuls les faits apaiseront les morts. »

Yamada: "Je rends hommage aux morts à ma façon. Vous le faites à votre manière, moi à la

mienne. C'est pourquoi je vais au sanctuaire Yasukuni..."

Okuzaki (livide de colère): "Tu dis que tu vas au Yasukuni? Tu penses donc que les âmes glorieuses du Yasukuni ont pu racheter leurs péchés, c'est ça, fumier?!

Madame Yamada (au moment où Okuzaki se jette sur Yamada, le faisant rouler par terre et

le prenant à la gorge) : "Il est malade. S'il vous plaît, s'il vous plaît, laissez-le tranquille. Si

vous continuez, vous allez le tuer... »

Okuzaki est un homme habité. Habité par l'intime conviction que non seulement les officiers et les sous-officiers du $36^{\mathrm{e}}$ corps du Génie ont tué Hashimoto et d'autres de leurs compagnons, mais qu'ils l'ont fait pour se nourrir de leur chair. Après l'agression d'Okuzaki, le sergent Yamada finit par tout avouer. Les officiers supérieurs, dit-il, ont en effet ordonné l'exécution d'hommes comme Hashimoto, car ils étaient mal-aimés au sein du régiment, ou qu'ils représentaient un poids pour celui-ci d'une manière ou d'une autre, et, après les avoir tués, ces mêmes officiers découpaient des lambeaux de leur chair, les faisaient cuire et les dévoraient. Les capacités respectives des deux hommes à se souvenir de cette époque, si différentes avant l'agression physique, se rejoignent à présent, donnant naissance à une mémoire partagée de l'enfer que représentait la Nouvelle-Guinée en 1945. Le neuvième chapitre de Yuki yukite shingun se referme sur Okuzaki réconfortant Yamada, et lui demandant avec un intérêt sincère comment va son infection urinaire.

La Nouvelle-Guinée a dû effectivement être l'enfer qu'Okuzaki décrit avec tant d'insistance. Le quartier général impérial envoya 160000 hommes mal préparés dans la partie orientale de l'île et seuls $6 \%$ d'entre eux survécurent; des dizaines de milliers d'hommes moururent de faim. Après-guerre, on dénonça unanimement les négligences et l'incompétence flagrantes de la préparation et de l'exécution de la campagne de Nouvelle-Guinée. Les autorités de la marine impériale avaient rassemblé des informations concernant les côtes de Nouvelle-Guinée, mais aucune concernant les réalités économiques et sociales de l'intérieur de l'île. Par exemple, ils ignoraient complètement que les habitants de la Nouvelle-Guinée étaient des chasseurs-cueilleurs, et que, par conséquent, il n'y aurait pas de champs, ni de récoltes pour subvenir aux besoins des combattants une fois que les lignes de ravitaillement seraient coupées. Et, pourtant ils savaient qu'elles seraient coupées, sans possibilité réelle d'un quelconque rétablissement étant données la distance effective entre le Japon et la Nouvelle-Guinée, et la mainmise grandissante des Américains sur le Pacifique. L'armée impériale, dont les hommes devaient débarquer puis combattre en Nouvelle-Guinée, n'avait même pas en sa possession une seule carte de l'île. Si le Japon voulait avoir une chance d'empêcher les avancées américaines et australiennes vers le Nord, les Japonais devaient occuper la Nouvelle-Guinée : c'est ainsi que résonnait le Haut Commandement impérial. Le général MacArthur était parti pour Brisbane après Pearl Harbor, et c'est de là, au nord de l'Australie, qu'il commandait la contre-attaque alliée.

5 Tanaka Hiromi, de l'Institut national de défense japonais, est l'un des seuls à avoir étudié la campagne de Nouvelle-Guinée de manière approfondie, et il se montre très cynique. D'après lui, cette campagne ne fut qu'un écran de fumée servant à masquer la défaite catastrophique subie par les Japonais dans cette même région, à Guadalcanal. Le Haut Commandement impérial n'avait pas envoyé un nombre suffisant de soldats, et ce peu de combattants, engagé sur plusieurs fronts, avait été submergé : au total, 20000 
Japonais ont été tués et 1000 fait prisonniers. Grâce à la campagne de Nouvelle-Guinée, l'armée impériale pouvait s'adresser à la nation en termes d'«avancées" et d'« assauts ", plutôt qu'en termes de " défaite » et d'« humiliation $»^{2}$. Toujours d'après Tanaka, le quartier général de l'armée impériale a effectivement abandonné à leur sort en Nouvelle-Guinée des dizaines de milliers d'hommes. Ils connurent un destin funeste. Rapidement encerclés, ils n'avaient le choix qu'entre lancer des attaques suicides dans les zones côtières, comme firent nombre d'entre eux, ou battre en retraite dans les jungles montagneuses où il fallait choisir entre la famine ou l'assassinat et le cannibalisme. Des archives australiennes nous confirment que ce dernier choix ne fut pas exceptionnel et que des soldats japonais, dans leur désespoir, tuaient et mangeaient des soldats australiens qu'ils avaient capturés, ainsi que des autochtones, en plus de leurs propres compagnons ${ }^{3}$.

La question qui constitue l'objet principal de cet article est de savoir de quelle manière on commémore au sanctuaire Yasukuni une campagne comme celle de NouvelleGuinée. Après tout, le site du Yasukuni est un lieu de deuil - et il se veut même lieu de deuil national - et en tant que tel, il est considéré comme un lieu de mémoire; car le deuil est impossible sans un acte de mémoire. Mon but ici est d'examiner deux des outils mnémoniques dont dispose le sanctuaire Yasukuni - l'ostentation et les rituels puis d'expliquer comment ces outils mnémoniques sont efficaces dans le cas de la Nouvelle-Guinée, et enfin de réfléchir à l'origine des comportements affichés vis-à-vis de l'acte de mémoire au Yasukuni ${ }^{4}$.

\section{L'ostentation}

7 Le premier outil mnémonique employé par le sanctuaire Yasukuni est l'ostentation, et dans ce cadre, le Yūshūkan 遊就館, le musée de la guerre situé dans l'enceinte du temple, adjacent au lieu de culte principal (haiden 抹殿), tient une place prépondérante. Je dis "prépondérante ", car l'ostentation ne se limite pas au musée. On trouve, par exemple, de nombreuses armes exposées dans l'enceinte du sanctuaire, y compris des répliques d'obus du cuirassé Yamato, ainsi qu'un énorme canon provenant du cuirassé Mutsu, qui fut récemment remonté des profondeurs de l'océan. On trouve également des bas-reliefs évoquant des scènes de guerre le long des murs qui mènent au lieu de culte. Pourtant c'est bien le Yūshūkan, dont la mission est d'« établir la vérité sur l'histoire moderne japonaise ", qui est le site principal. Sur ses murs sont exposés des panneaux relatifs aux différents champs de batailles de la Guerre du Pacifique, y compris la Nouvelle-Guinée.

8 On pouvait difficilement s'attendre à ce que le panneau concernant la Nouvelle-Guinée représente ce qu'y ont vécu Okuzaki, Yamada et Hashimoto, et cela rend d'autant plus fascinant ce qui y est effectivement représenté. On y trouve une photo de l'amiral Yamamoto Isoroku 山本五十六 (1884-1943) plongé dans la lecture d'une carte; selon toute apparence, il est en train de régler les détails de cette fameuse campagne qui n'a en fait jamais été réellement organisée (du reste, ce personnage est également le responsable du désastre de la bataille de Midway). Ses jumelles, exposées dans une vitrine, semblent également mettre l'accent sur sa clairvoyance, une clairvoyance qui fit pourtant cruellement défaut lors de la campagne de Nouvelle-Guinée. On y trouve également des morceaux du fuselage de l'avion qui le conduisait sur le théâtre des opérations et qui s'écrasa dans la jungle de Nouvelle-Guinée en 1943, lui ôtant la vie 
avant que l'enfer de cette campagne n'ait véritablement commencé. On peut aussi y voir la casquette du Lieutenant de Marine Yasuda, et un télégramme que celui-ci envoya à Yamamoto avant de mener ses hommes dans une attaque suicide. Figure en outre à cet endroit un commentaire nous invitant à méditer sur « l'humanité dont les Japonais ont fait preuve en Nouvelle-Guinée, humanité qui a légué à la postérité de nombreux récits devant être racontés: les attaques-suicide à Buna, la tragédie de Danbir, et le passage des Monts Sarawaket. "

Certains points sont à souligner :

- Il n'est pas fait mention ici de l'incompétence des officiers, de la famine, de la maladie ou du cannibalisme, ce qui n'est guère étonnant. On a préféré substituer à ces évènements les notions de planification, de clairvoyance, d'endurance et d'héroïsme.

- Ces récits héroïques - et il ne fait aucun doute que des hommes tels que le lieutenant Yasuda firent preuve d'un courage remarquable - ne concernent que des officiers et des sousofficiers ; aucun ne mentionne de simples soldats.

- Il y a encore quelque chose de curieux à propos de ce panneau, et de tous les autres panneaux du musée Yūshūkan: on ne trouve nulle part de représentation, ou même de simple évocation, de l'ennemi contre lequel combattaient Yamamoto, Yasuda et les autres. Grâce à cette absence d'ennemi, le panneau concernant la Nouvelle-Guinée entrave toute possibilité d'évoquer la défaite et les mauvaises actions qui y ont été commises ${ }^{5}$.

Il est évident que pour des hommes comme le sergent Yamada, les pièces concernant la Nouvelle-Guinée exposées au Yūshūkan sont d'un grand réconfort, ou du moins était-ce le cas avant sa rencontre avec Okuzaki. En tout cas, elles ne remuaient pas les souvenirs horribles qu'il avait réussi à enfouir au plus profond de sa mémoire. Pour ces mêmes raisons, il est probable que les rites du Yasukuni, auquel il dit avoir participé dans le bâtiment principal du sanctuaire, ont profondément soulagé Yamada. Ceci nous amène à aborder le second outil mnémonique employé par le sanctuaire Yasukuni : l'accomplissement de rites.

\section{L'accomplissement de rites}

11 Les rites sont la raison d'être du sanctuaire Yasukuni et de ses desservants. Ces derniers accomplissent deux types de rites: des rites dynamiques de consécration qui transforment l'esprit d'un défunt en kami ou en eirei 英霊, une âme héroïque, et des rites de propitiation, connus sous le nom de ireisai 慰霊祭, au cours desquels des desservants font des offrandes de nourriture et des prières aux kami du panthéon du sanctuaire Yasukuni ${ }^{6}$. En échange, les kami accordent leur bénédiction aux vivants. De tels rites ont lieu tous les jours, matin et soir, ce qui montre que le sanctuaire Yasukuni est d'abord et avant tout un lieu rituel. L'accomplissement de rites est évidemment un élément fondamental dans l'élaboration d'une mémoire collective.

Ce qui est saisissant dans l'échange qui a mené à l'agression de Yamada par Okuzaki, c'est son élément déclencheur; les deux hommes n'étaient pas d'accord sur ce qui devait apaiser les âmes des morts. Okuzaki insista sur le fait que la proclamation de la vérité et la demande de pardon étaient des prérequis indispensables à cet apaisement. Le sergent Yamada rétorqua qu'il n'avait à s'excuser de rien et lui fit remarquer qu'il accomplissait régulièrement des actions de propitiation pour ses compagnons disparus, en assistant à des rites au Yasukuni. C'est au moment où il a prononcé le mot «Yasukuni » qu'Okuzaki a bondi, se jetant sur lui, et pendant qu'il le battait et tentait 
de l'étrangler, il hurlait: "Tu dis que tu vas au Yasukuni? Tu penses que les âmes héroïques du Yasukuni ont pu racheter leurs péchés, c'est ça, fumier ?! »

Okuzaki haïssait le Yasukuni et il confia un jour à Hara Kazuo, le réalisateur du documentaire Yuki yukite shingun, qu'il pensait lancer une attaque contre le sanctuaire pendant la Célébration de printemps, armé d'un sabre court. La Célébration de printemps est un des deux événements majeurs du calendrier du Yasukuni, et il se distingue des autres par la présence d'un émissaire impérial. Okuzaki déclarait avoir l'intention d'abattre l'émissaire impérial ainsi que les desservants et d'autres personnes. Il est possible d'imaginer pourquoi Okuzaki exécrait le Yasukuni à ce point. C'était un homme obsédé par la vérité et sa proclamation, par le devoir et la responsabilité, et par la commémoration des morts. Il savait que les rites de consécration et de propitiation de ses camarades disparus et de ses supérieurs avaient valeur d'absolution: les rites du Yasukuni les absolvaient de toute responsabilité et niaient ce qu'Okuzaki savait être la vérité. Je suppose en effet que c'est cela qu'Okuzaki ressentait vis-à-vis du Yasukuni, mais on ne peut en être certain. En revanche, une chose est sûre : ce sont ces mêmes sentiments qu'lida Susumu éprouve à l'égard du sanctuaire. Iida était sous-officier en Nouvelle-Guinée; il fut traduit en justice devant un tribunal de guerre au Japon et reconnu comme criminel de guerre de catégorie B pour sa conduite pendant le conflit. Récemment, lors d'une émission spéciale de la NHK sur le Yasukuni, Iida évoqua de manière détachée la volonté du sanctuaire d'" apaiser " et d'«honorer » les " âmes héroïques» des soldats morts au combat ${ }^{7}$. Puis il reconnut devant les caméras: «En effet, ce sont de belles paroles. Elles effleurent une corde sensible chez les endeuillés, chez les compagnons d'armes, et chez de nombreux Japonais d'ailleurs. Mais, du point de vue de quelqu'un qui a combattu pendant cette guerre, il est nécessaire de poser la question : ces hommes méritent-ils les honneurs en tant que héros morts pour la patrie ? Je ne le pense pas.»

Iida insista sur le fait que 100000 soldats et officiers japonais moururent de faim en Nouvelle-Guinée, et poursuivit :

En tant que simples soldats, qui méprisions-nous? Les commandants qui avaient planifié la campagne de Nouvelle-Guinée. Si on y réfléchit bien, des expressions telles que "les âmes héroïques des hommes morts au combat " ne servent en fait qu'à éluder la question des responsabilités.

Iida conclut ainsi : «Le [refus] de ces hommes [à accepter leurs responsabilités] est inacceptable. »

16 Le lien entre la mémoire et les rites du Yasukuni, qui rendent hommage à tous les disparus, sans distinction, en tant qu' " âmes héroïques », est assez clair. Néanmoins, il mériterait d'être explicité. Les rites de propitiation, en honorant l'ensemble des disparus, rendent hommage à un nombre impressionnant de Japonais partis au combat; plus de deux millions d'entre eux ont donné leur vie pour le Japon et pour l'empereur. Ils commémorent les disparus comme des hommes ayant incarné les vertus sacrées : loyauté, patriotisme, don de soi. Ils commémorent les disparus en glorifiant leurs actes, et non en pleurant leur mort tragique. Ils commémorent la guerre comme un acte noble et riche de sens, même si elle s'est achevée par une défaite.

Qu'oublie-t-on dans ce rite de commémoration?

- Qu'outre les personnes qui sont mortes en faisant preuve d'un courage extraordinaire, des milliers sont mortes de faim ou de maladie et d'autres ont été abattues pour être mangées. 
- Que par définition toutes les guerres - et la campagne de Nouvelle-Guinée plus que toute autre peut-être - sont violentes et sordides.

- Que ces hommes et ces femmes qui incarnaient véritablement la loyauté, le patriotisme et le don de soi étaient des victimes du militarisme japonais, et de son incompétence. Leur mort fut un gâchis.

\section{Réprimer le traumatisme}

18 Il faut souligner que la stratégie utilisée par le Yasukuni pour aborder la campagne de Nouvelle-Guinée, que ce soit dans les expositions du musée Yūshūkan ou dans les rites de propitiation, n'est pas propre à ce sanctuaire, ni même au Japon. Cependant, le déploiement de telles stratégies visant à réprimer les souvenirs de guerre trop douloureux n'est pas toujours aussi flagrant. Ce sont précisément ces stratégies qu'Éric Santner appelle « fétichisme narratif ». Santner écrit :

J'entends par "fétichisme narratif» l'élaboration et le déploiement d'un récit, dont le but conscient ou inconscient est d'effacer toute trace du traumatisme ou de la perte qui était à l'origine même de ce récit. ${ }^{8}$

Le fétichisme narratif, qui pour Santner est synonyme de la "production de mythes ", permet de débarrasser une personne du fardeau que représente la reconstruction de sa propre identité dans des conditions post-traumatiques ; car dans le fétichisme narratif, le « 'post' est repoussé indéfiniment ».

Je pense être suffisamment conscient des risques qu'il existe à appliquer de manière trop simpliste une théorie à la stratégie mémorielle extrêmement complexe que met en œuvre le sanctuaire Yasukuni ; il me semble néanmoins que les observations de Santner proposent des éléments de réponse. La consécration de tous les disparus de NouvelleGuinée sans distinction, leur transformation en kami et en âmes glorieuses, ainsi que les choix judicieux des objets et photos exposés sur et autour des panneaux sur la Nouvelle-Guinée dans le musée Yūshūkan semblent constituer une sorte de fétichisme narratif. La mort de deux millions de Japonais, l'humiliation de la défaite puis l'occupation, la démilitarisation conduite par l'occupant, la Constitution imposée par l'occupant qui amena la paix et la prospérité japonaise de l'après-guerre, toutes ces sources de traumatismes sont soigneusement effacées dans le processus de commémoration du sanctuaire.

\section{En conclusion}

21 Notre bref examen de ce que fait le Yasukuni dans le domaine de la mémoire, et les raisons pour lesquelles il le fait, nous permettent de conclure que ce sanctuaire ne peut constituer de manière satisfaisante le site national de soutien à l'État. Son incapacité flagrante à commémorer le passé de manière complexe et fragmentée, sa persistance à présenter une histoire lisse et sans nuances, en d'autres termes son amnésie volontaire, suscitent un nouveau "problème du Yasukuni ", qu'il faut ajouter à ceux déjà existants : le débat constitutionnel soulevé par la protection que lui ont accordée les chefs du gouvernement et celui, symbolique, posé par les rites de consécration et de propitiation d'hommes reconnus comme étant des criminels de guerre de catégorie A. Bien sûr, le sanctuaire Yasukuni est une entité privée au regard de la loi, et non, malgré ses prétentions, un site national. Par conséquent, il possède une entière liberté 
d'action. Mais lorsque l'État affiche, par des visites du Premier ministre ou de membres du Cabinet, la protection qu'il accorde au sanctuaire, il agit comme si la Constitution lui importait peu. L'État semble en outre prendre ses distances par rapport aux verdicts rendus par le Tribunal militaire international pour l'Extrême-Orient (le Procès de Tōkyō) et accorder foi à la version de l'histoire que propose le Yasukuni.

Il faudrait créer un autre site commémoratif. Sans lui, la cicatrisation définitive des blessures de guerre n'aura jamais lieu. La création d'un nouveau site commémoratif est à mon avis une option plausible, pour au moins trois raisons. La première raison est qu'un tel site, débarrassé comme il se doit du musée, des desservants shintō et de leurs rites, pourrait rendre possible la conception d'un phénomène de mémoire plus complexe, et proposer une alternative à ce fétichisme narratif qui a cours au Yasukuni. La seconde raison, qui découle de la première, est que favoriser une mémoire plus complexe, éliminer le fétichisme narratif qui gomme les traumatismes sont des conditions nécessaires pour que le deuil puisse enfin s'accomplir sans qu'interfèrent des questions politiques et morales. Un tel site rendrait également possible une réflexion sur le passé et un travail de deuil commun que le Premier ministre japonais, le Premier ministre britannique, et pourquoi pas, le Premier ministre chinois mèneraient main dans la main. La troisième raison en faveur de la création de ce nouveau site est qu'un deuil, ainsi débarrassé de ce qui le parasite, favoriserait la construction d'une identité post-conflit, qui ne se définirait pas par rapport à la guerre.

De mon point de vue, les chefs de gouvernement japonais se doivent de présenter leurs hommages aux hommes et aux femmes qui - volontairement ou non - ont donné leur vie pour le Japon lors de campagnes perdues d'avance telles que celle de NouvelleGuinée. Un nouveau site rendrait ces hommages acceptables moralement, et possibles d'un point de vue diplomatique.

\section{NOTES}

1. Le titre de ce documentaire, traduit en anglais, est "The Emperor's Naked Army Marches On" (L'armée nue de l'empereur poursuit sa route). Il fut primé à de nombreuses reprises, entre autres, au Festival international du Film de Berlin (1987), au Blue Ribbons Award (1987), au Festival international du Film de Rotterdam (1988) et au Festival du Film de Yokohama (1988) [NDT].

2. Voir Tanaka Hiromi, "The Pacific War and New Guinea" (La Guerre du Pacifique et la NouvelleGuinée) sur le site: http://ajrp.awm.gov.au/ajrp/ajrp2.nsf/WebI/Chapters/\$file/Chapter1.pdf? OpenElement.

3. Sur ce point, consulter Tanaka Yuki, Hidden Horrors: Japanese War Crimes in World War 2 (Horreurs cachées: les crimes de guerre japonais pendant la Seconde Guerre mondiale), Colorado, Westview, 1996, p. 111-134.

4. J'ai déjà étudié (certes, dans une perspective différente), les outils mnémoniques que sont les rituels et l'ostentation. Voir Breen, "Yasukuni and the Loss of Historical Memory" (Yasukuni ou la perte de mémoire historique), in John Breen (dir.), Yasukuni, the War Dead and the Struggle for Japan's Past (Yasukuni : les disparus de la Guerre du Pacifique et le combat pour la 
réappropriation du passé), London, Hurst, 2007. Dans cet ouvrage, j'aborde l'utilisation par le sanctuaire de textes visant à l'élaboration de la mémoire.

5. Sur l'absence de l'ennemi au Yūshūkan, voir Breen, Yasukuni and the Loss of Historical Memory, op. cit.

6. Pour une discussion approfondie sur ces deux types de rituels, voir Breen, «The Dead and the Living in the Land of Peace: a Sociology of the Yasukuni Shrine » (Les vivants et les morts au pays de la paix : étude sociologique du sanctuaire Yasukuni), Mortality 9.1, 2004, p. 76-93, notamment p. 77-81.

7. «Sengo rokujū nen : Yasukuni mondai wo kangaeru» 戦後60年 靖国問題を考える (Soixante ans après la fin de la guerre : réflexions sur la question du Yasukuni), programme NHK Supesharu du 14 août 2005.

8. Eric Santner, "History beyond the Pleasure Principle: some Thoughts on the Representation of Trauma" (L'Histoire au-delà du plaisir : pensées concernant les différentes manifestations d'un traumatisme), in Saul Friedlander (dir.), Probing the Limits of Representation: Nazism and the Final Solution (Sonder les limites de la représentation : le nazisme et la solution finale), Cambridge, Cambridge University Press, 1992, p. 144.

9. Concernant l'ouverture d'un autre site et les contre-arguments, voir Breen, Yasukuni and the Loss of Historical Memory, op. cit.

\section{RÉSUMÉS}

L'exemple de la commémoration qui est faite de la Campagne de Nouvelle-Guinée (1942-1945) au Yasukuni illustre son incapacité à commémorer le passé, et appelle à la création d'un nouveau site.

The New Guinea campaign (1942-1945) commemorative ceremony illustrates the inability of the Yasukuni shrine to commemorate the past.

\section{INDEX}

Keywords : Historiography, History, Japon -- War Crime Trials, Memory, Orientalism, War of the Pacific (1939-1945), Yasukuni mondai

Index chronologique : guerre du Pacifique (1941-1945)

Mots-clés : crimes de guerre -- procès, guerre de mémoire, orientalisme, sanctuaire Yasukuni Index géographique : Nouvelle-Guinée

キーワード : sensō hanzai 戦争犯罪, kioku 記憶, orientarizumu オリエンタリズム, Yasukuni jinja 靖国神社, Nyū Ginia ニューギニア, Taiheiyō sensō 太平洋戦争 (1941-1945), shigakushi 史 学史, rekishi 歴史

Thèmes : histoire, historiographie 